\title{
What Are Auditory Objects?
}

\author{
MATTHEW NudDS
}

The tenth variation of Bach's Goldberg Variations is a short four-voice fugue. It begins with a subject in the bass (on $\mathrm{G}$ below middle $\mathrm{C}$ ) which is answered after four bars in the tenor; the soprano voice begins after a further four bars, and the final alto voice begins four bars later. When played at a normal tempo one doesn't hear the variation as a sequence of notes in the order in which they are played. As each new voice begins its notes are heard as a distinct melody - a separate voice parallel to the other voices - so that after twelve bars one hears four parallel melodies playing simultaneously. It is possible to attend selectively to each melody, but difficult to attend to more than one simultaneously; instead, when one attends to one melody it is heard against a background of the others. Although one can hear the order of and temporal relations between the notes that make up each melody, it is impossible to hear the order of the notes that make up the variation as a whole, and impossible to hear the temporal relations between the notes as they are actually played. ${ }^{1}$

This is an example of auditory grouping. We experience a sequence of notes as a number of separate groups or streams each of which is made up of only some notes in the sequence. This kind of grouping is central to our experience of many kinds of music, but grouping in music is the result of a process that occurs in, and is essential to, auditory perception generally - or so, at least, I shall argue.

\section{Which objects are auditory objects?}

Sounds are those objects of experience that can be characterised in terms of their loudness, pitch, and timbre. We can regard any sound as an auditory object, but I am going to use the term 'auditory object' to mean those temporally extended sequences of sounds that are experienced as a group in the way that we experience the notes of each melody as grouped. These sequences may or may not be composed of discrete, countable, sounds; in what follows I will talk of parts of a sequence and mean either the discrete sounds of a sequence or simply the temporal parts of a sequence that are

\footnotetext{
${ }^{1}$ It is impossible, at least, for someone who is not already familiar with the musical score, i.e. with the order in which the notes are played.
} 
not experienced as discrete. ${ }^{2}$ By reserving my use of the term 'auditory object' for sequences of sounds experienced as a group I don't mean to imply that there is some fundamental difference between auditory objects and other sounds. There is not. It is simply that the issues that I want to discuss arise most clearly for grouped sequences of sounds.

When we experience two or more sequences simultaneously (as we do with Bach’s Goldberg Variation) we experience parts of each sequence belonging with other parts of the same sequence, but as not belonging with parts of the other sequence. That parts are grouped together has consequences for our perceptual experience. Many of the properties and features awareness of which constitute our auditory experience are properties or features of groups of sounds. For example, we experience melodic, rhythmic, or other patterns amongst parts of a grouped sequence, but not amongst parts that belong to different groups; we can often tell the order of parts in a grouped sequence, but not the order of parts that belong to different groups; ${ }^{3}$ and we can attend to one group to the exclusion of another, but not to two or more different groups simultaneously, nor can we simultaneously attend to two or more parts that belong to different groups. What I am calling auditory objects are those sounds which are experienced as grouped and so as having properties of this kind. It is these objects - objects that we can pick out in our auditory experience - that I am going discuss in this paper.

Not all sequences of sounds are auditory objects (as I am using the term) because not all sequences of sounds are experienced as a group. When we experience a sequence as a group we experience the different parts of the sequence as belonging together. When the parts of the sequence are not discrete parts we experience the sequence as a single, continuous, sound; when the parts of the sequence are discrete our experience still seems to be of a single thing - a single auditory object - but our experience is discontinuous: we experience individual sounds as parts of a single auditory object, our experience of which is seems somehow interrupted. The experience of hearing such an auditory object is analogous to the experience of seeing an object through the railings of a fence: the parts of the object we see through the

\footnotetext{
${ }^{2}$ There is no sharp boundary between the two kinds of sequence. Although different questions arise for each, as far as the issues that I discuss in this paper are concerned the differences don't matter.

${ }^{3}$ It's not always possible for listeners to perceive the order of elements in a group, but listeners can discriminate groups composed of different orderings of the same elements: the order of elements in a group determine how that group sounds; elements in other groups don't.
} 
gaps in the fence look to be parts of a single object of which we have an interrupted view. ${ }^{4}$ The analogy might strike you as a poor one. After all, in the visual case there is a single object we see, and we see the fence that interrupts our view of it, whereas in the auditory case the sounds we hear are in fact discontinuous, and we are not aware of anything interrupting our hearing them. But the problem in both cases is the same: it is the problem of explaining what it is to experience parts of what appears to be a single object - be it a visual or an auditory object - as belonging together as parts of that object. In the case of vision the problem is tractable: the parts are parts of a material object, so the problem is that of explaining what it is to see parts as parts of a material object. In the auditory case, things are not so straightforward. The parts are parts of an auditory object, but if an auditory object just is what we experience when we experience sounds as a group then it's not clear how we can explain what it is to experience sounds as a group in terms of hearing them to be parts of, or as belonging to, a single auditory object. A satisfactory explanation of what it is to hear sounds as parts of a single auditory object must do two things. First, it must give some account of what our experience of auditory objects is an experience of - it must tell us what auditory objects are 'in the world' independently of our experience of them - and second, it must give an account of the content of our experience of auditory objects it must, that is, explain what it is to experience a sequence of sounds as parts of a single auditory object. These two questions are related, but an answer to one is not an answer to the other. ${ }^{5}$

\footnotetext{
${ }^{4}$ The auditory grouping that I discuss in this paper should be distinguished from auditory perceptual restoration effects such as the continuity illusion (for a description of such effects and the circumstances in which they occur, see Warren (1999, ch.6)). Perceptual restoration effects concern the perception of auditory stimuli - what we might loosely call the sound itself - whereas auditory grouping concerns the perception of the source of the sound. Auditory grouping is therefore analogous to the visual perception of occluded surfaces and objects, whereas auditory perceptual restoration is not.

${ }^{5}$ There is a parallel here to the account we should give of the visual perception of causation. People can visually discriminate objects that appear causally related from objects that do not appear causally related. In order to determine the circumstances in which objects appear to be causally related, Michotte (1963) conducted a series of experiments in which subjects were shown the interactions of pairs of objects. He discovered that if subjects saw, for example, a moving object X collide with a stationary object $\mathrm{Y}$ which then started to move, they had the impression that X caused $\mathrm{Y}$ to move; if, however, there was a delay before object Y started to move, subjects no longer had the impression that $\mathrm{X}$ caused Y to move. Subjects had impressions of causation even when these 'objects' were patches of light projected on a screen. We can ask two questions about the subjects' visual experience in such cases. First, what relation 'in the world' is their experience of causation tracking, what is it an experience of? Second, does their visual experience represent the relation between objects as one of causation? Even if their experience of causation tracks causation in the world, it wouldn't follow that they experience it as such; and even if their experience represents causation, it wouldn't follow that there is causation in the world since their experience may be non-veridical.
} 


\section{Why do we experience auditory objects?}

It is sometimes suggested that we can explain what it is to hear sounds as parts of a single auditory object by appealing to principles of auditory experience analogous to Gestalt principles in vision. ${ }^{6}$ To do so is to explain auditory objects in a purely auditory way, where an explanation of auditory objects is purely auditory if it explains them only in terms of the auditory or sensory properties of sounds. The following examples illustrate this kind of explanation.

Auditory grouping can occur with simple sequences of tones. When listeners are played a sequence of six tones - three from a high-pitch range and three from a low pitch range - what they hear depends on the rate at which the tones are played. When they are played slowly listeners hear the tones in the order in which they are played; when they are played fast (at a rate of ten per second) they hear two parallel sequences, one of repeating low pitch tones and the other of repeating high pitch tones. ${ }^{7}$ When listeners are played a sequence of tones that alternates between a rising a falling trajectory they do not hear the sequence as two linear trajectories that cross at the midpoint; they hear the tones as grouped into two sequences made up of the higher pitch and the lower pitch tones. However, if the tones that make up the rising trajectory are given a different timbre from those that make up the falling trajectory, two crossing sequences are heard.

In these two examples, sounds are experienced as organised into two groups and the groups are heard as distinct. Each sound is experienced as belonging to one group but not the other. What explains why the sounds are experienced as grouped in the way they are? Altering the properties of the sounds, or the speed at which they are played, changes the way they are grouped; it might seem, therefore, that grouping is determined by properties of the sounds. For example, sounds that are similar and temporally proximal tend to be experienced as grouped together; there are different dimensions of similarity, and sounds may be similar in virtue of their pitch or in virtue of their timbre with - as the second example demonstrates - timbre contributing more to similarity than pitch. So it might seem that we can give an account of auditory

\footnotetext{
${ }^{6}$ For example, Bregman (1990, ch.2); Deutsch (1999).

${ }^{7}$ This example is described in more detail in Bregman and Campbell (1971); and Bregman (1990, p.17).
} 
objects in a purely auditory way, in terms of properties of sounds and the relations between sounds. According to this account, auditory objects are sequences of sounds that are related to one another in various ways - that have a similar pitch, are temporally proximate, and so on - and our experience of an auditory object just is an experience that represents a number of sounds as having these properties and standing in these relations to each other.

\section{What's wrong with purely auditory explanations?}

When we experience a sequence of sounds as grouped each sound is experienced as belonging together with other sounds - as being parts of a single auditory object. What explanation can the purely auditory account give of what it to experience sounds as belonging together as parts of a single auditory object?

The explanation cannot just be that to experience a sequence of sounds as grouped is to experience a sequence of sounds that satisfy the principles of grouping a sequence of sounds that, say, have the same timbre. To say that is just to say that we experience each sound of the sequence as having a timbre which is the same as the others. That adds nothing to the description of the content of the experience, and so it doesn't explain what it is to experience them as grouped. A defender of the pure auditory account might claim that we don't just experience sounds as having the properties in virtue of which they satisfy the principles of grouping, but that we experience them as satisfying those principles: that we experience sounds with the same timbre as having the same timbre. To experience an auditory object, according to this suggestion, is to experience a sequence of sounds as having the same timbre (or as being related along some other relevant dimension of similarity).

An initial problem with this suggestion is that it's not clear what it means to say that we experience a sequence of sounds as similar to each other if that doesn't simply mean that we experience them as having the properties in virtue of which they are similar. But, for the sake of argument, suppose that some explanation of that is possible. The resulting account still does little more than describe the phenomenon we wanted to explain: it does little more than point out that auditory objects are composed of sounds that are, in certain ways described by the principles of grouping, similar to each other, and that we experience them as such. It doesn't tell us what it is in general to experience a sound as part of an auditory object. In each case, the pure 
auditory account can point to some dimension (or dimensions) of similarity sameness of timbre, similarity of pitch, temporal proximity, and so on - that we experience the sounds as having to each other, but it doesn't explain what is common to all these different particular explanations. For example, in one case we might experience a sound as part of an auditory object in virtue of experience is as having the same timbre as those other sounds; in another case we might experience a sound as part of an auditory object in virtue of being harmonically related; the explanation is different in each case; we have no explanation, therefore, of what it is in general to experience a sound as part of an auditory object.

The problem is that the purely auditory account does not explain why we experience as grouped sounds that satisfy the principles of grouping - it doesn't explain why those principles of grouping are true and not others. The principles tell us in what circumstances we experience sounds as grouped, but not why we experience sounds as grouped in those circumstances. They do not, therefore, provide a satisfying explanation of our experience of auditory objects. In what follows I explain why we experience sounds as grouped in just those circumstances described by the principles of grouping, and I use that explanation to provide an account both of what auditory objects are, and of what we experience them as being.

We experience sounds as grouped - and so experience auditory objects - as a consequence of the way the auditory system functions; so to understand why we experience sounds as grouped in the circumstances described by the principles of grouping we need to understand how auditory perception functions.

\section{Auditory Function}

What is the function of auditory perception? Auditory perception, like vision, functions to tell us about objects in our environment; it does so by detecting disturbances in the air produced by those objects, and by events involving them. Although it is perhaps obvious that vision functions to tell us about objects, that auditory perception does so too is not obvious. I shall begin by outlining an account of auditory function that justifies that claim (by showing how it can have that 
function), and then go on to say something about the consequences it's having that function has for an account of auditory objects. ${ }^{8}$

It is often assumed that the purpose or function of auditory perception is to tell us about sounds. Although auditory perception does tell us about sounds, that is not its function. Imagine that you are woken up in the middle of the night by a strange sound. As you lie there, listening, you can attend to your experience in two ways: you might attend to the sound itself, focussing on its attributes - its pitch, timbre, and loudness - but it is more likely that you will attend to what is making the sound: that it is the sound of footsteps, that it is coming from the stairwell, and that they seem to be coming closer. When people are asked to describe what they hear (in psychoacoustics experiments, for example) they are often encouraged to attend to their experience in the first way: to describe the sensory attributes of the sounds they hear in abstraction from whatever it was that produced the sounds. ${ }^{9}$ They may be helped by being played harmonically simple sounds produced by a tone generator, sounds which develop little over time and which have little or no ecological significance. There is little to describe about such an experience over and above the sensory qualities of the sounds. The majority of the sounds we hear are not like that, and most everyday listening is of the second kind: we attend to the apparent sources of the sounds we hear and listen to the things going on around us: to the objects and events that produced the sounds. In most everyday listening we are concerned with properties and attributes of the sound producing events and objects, and with the environment in which they occur, and not with properties of the sound itself. ${ }^{10}$

Although relatively little investigation has been done to determine how good we are at perceiving and recognising sound sources, that which has been done has found that we are (perhaps surprisingly) good at both. There is evidence that we are capable of recognising very specific characteristics of the events and objects we

\footnotetext{
${ }^{8}$ The account is this section draws on the more detailed discussion and defence given in Nudds (forthcoming).

${ }^{9}$ When they do this, listeners adopt what Gaver (1993) calls a 'musical' and Scruton (1987, pp.2 ff.) an 'acousmatic' attitude to what they hear.

${ }^{10}$ In what follows I am going to talk about the perception of what may be labelled 'ecological' sounds and their sources - those sounds produced by naturally occurring events or various kinds (and excluding sounds produced by musical instruments and recorded sounds). Ecological sounds themselves, rather than their sources, are not very interesting or informative; indeed for many ecological sounds it is actually rather difficult to attend to the sound rather than to the source of the sound and we are poor at describing the character of the sound. It is easy to overlook the nature of ecological sound perception because we have become so used to hearing artificially produced sounds.
} 
hear. ${ }^{11}$ We are, for example, very good at recognising what kind of object or event produced a sound. Listeners who were played recordings of different size jars and bottles falling to the ground and either bouncing or breaking and were asked which kind of event - a bouncing or a breaking - they heard were almost always correct. ${ }^{12}$ When asked to identify thirty common natural sounds in a free identification task sounds such as those produced by clapping, tearing paper, and footsteps - listeners recognised source events very reliably; they described the sounds in terms of the objects and events which caused them, and only described the sensory qualities of sounds whose source events they could not recognise. ${ }^{13}$ In a similar experiment in which seventeen sounds were played, listeners were asked to identify what they heard. They nearly always described the sounds in terms of their sources, and were surprisingly accurate. Several participants could readily distinguish the sounds made by someone running upstairs from those of someone running downstairs; others were correct about the size of objects dropped into water; and most could tell from the sound of pouring liquid that a cup was being filled. Some sounds - such as the sound of a file drawer being opened and closed - were difficult to identify, but the listeners' descriptions revealed what might be regarded as basic attributes of what was heard: "several people said the file drawer sounded like a bowling alley, both of which might be described as 'rolling followed by impact(s)'”, 14 Further investigation is likely to reveal many more examples.

As well as recognising the sources of sounds we can perceive their properties. We are, for example, able to perceive the trajectory of an approaching sound source, ${ }^{15}$ and the time to contact - that is, the time at which we will collide - with a sound source that is moving towards us. ${ }^{16}$ We are good at hearing whether an invisible

\footnotetext{
${ }^{11}$ For a recent survey of much of this evidence, see Carello et al. (2005). Compare what I describe here to accounts of visual object recognition, which has been studied in great detail and is widely understood to be a perceptual phenomenon with the results of the process of object recognition entering into the content of visual experience. I know of few equivalent studies of object recognition in auditory perception, but see McAdams (1993) and Peretz (1993).

${ }^{12}$ Listeners' success rate was 99\%; see Warren and Verbrugge (1984).

${ }^{13}$ The success rate was about 95\%; see VanDerveer (1979).

${ }^{14}$ Gaver (1993a, p.12). It is plausible to suppose that recognising such events involves the perception of simpler, more fundamental, properties of events and that such properties may be perceived even when the event is not recognised. In much the same way visual recognition of an object as, for example, a television, involves perceiving the object as having more fundamental properties such as size and shape which it may be perceived as having even when it is not recognised as a television.

${ }^{15}$ Neuhoff (2004).

${ }^{16}$ Schiff and Oldak (1990).
} 
object making a noise is within reach; ${ }^{17}$ and we are able to hear just as well as we can see whether a gap between a sound source and a vertical surface is wide enough to pass through. ${ }^{18}$ We can identify the material composition of an object from the sound of an impact, ${ }^{19}$ and perceive the force of the impact. ${ }^{20}$ More surprisingly, perhaps, we are able to distinguish objects on the basis of their geometrical properties. When differently shaped - circular, square, and triangular - flat steel plates of the same mass and surface area were suspended and struck by a steel pendulum released from a fixed location, listeners sitting behind a screen were able to classify the shapes at a level well above chance. A similar experiment was conducted with rectangular steel plates of different proportions and dimensions chosen so that all were equal in mass and surface area. Listeners had to respond by adjusting lines to provide a visual match for the height and width of the plate. Although they were given no other information about the size of the object, the actual linear dimensions of the plates accounted for $98 \%$ of the variance in the listeners' responses. ${ }^{21}$ Similarly, when listeners were asked to indicate the lengths of cylindrical rods dropped to the floor, the actual length of the rods accounted for $95 \%$ of the variance in perceived length. ${ }^{22}$

Given that we can perceive and recognise the sources of the sounds we hear, it is plausible that auditory perception functions to tell us about those sources, and that in addition to representing sounds, our auditory experience represents the sources of sounds and their properties. For such a suggestion to be plausible, we need to explain how auditory perception could have this function. We should begin with how sound sources produce sounds. Sounds can be produced by many different kinds of things liquids, solid objects, strings, air movement - and in many different ways, but for simplicity I am going to consider only material objects. Material objects produce sounds when they are struck, tapped, scraped, broken or otherwise caused to vibrate.

We often picture a vibration as a single sine wave. Not even something as simple as a plucked string vibrates in such a simple way. The vibration of a plucked

\footnotetext{
${ }^{17}$ Carello et al. (1998).

${ }^{18}$ Russell and Turvey (1999).

${ }^{19}$ Wildes and Richards (1988).

${ }^{20}$ Freed (1990).

${ }^{21}$ The plates were a square $(482 \mathrm{~mm})$, a medium rectangle $(381 \mathrm{~mm} \times 610 \mathrm{~mm})$, and a long rectangle (254mm x 914mm), the width indicator ranged from 0 to $2.5 \mathrm{~m}$, and the height indicator from 0 to $1.5 \mathrm{~m}$. Although listeners' relative scaling of the plates was accurate, the perceived dimensions were underestimates of actual dimensions, ranging from $252 \mathrm{~mm}$ to $445 \mathrm{~mm}$ for an actual range of $254 \mathrm{~mm}$ to 914mm (Kunkler-Peck and Turvey 2000).

${ }^{22}$ Carello, et al. (1998).
} 
string is complex; it comprises a number of simple vibrations at frequencies which are integer multiples of the lowest, or fundamental, frequency of the vibration. ${ }^{23}$ Any complex vibration is equivalent to a number of simple frequency components superimposed on each other; that means we can represent any complex vibration as a pattern of individual frequency components. Objects vibrate along a greater number of dimensions than strings and consequently their vibrations are more complex and so composed of a greater number of frequency components. What's important for our understanding of auditory perception is that the particular pattern of frequency components produced by a material object when it vibrates is determined in a law-like way by both the physical nature of the object and the nature of the event that caused it to vibrate. For example, the shape and size of the object determine the lowest frequency of its vibration and what harmonics are present. The overall amplitude of the vibration is determined by the force that initially deforms the object, but because objects are not linearly elastic the amplitude of individual frequency components varies with the force of the initial deformation. The spectral composition of the vibration therefore changes according to how hard the object was struck. ${ }^{24}$ Vibrating objects lose energy over time and their vibration decays. The rate of decay of different frequencies components - and so changes in the spectral composition of the vibration over time - is determined by the material of which the object is composed.

The pattern of frequency components that comprise the vibration of an object and the way that pattern changes over time is determined by the nature of the object and the nature of the events that caused it to vibrate. That pattern and the way it changes therefore embody information about the object that produced the vibration and the event that caused it to vibrate. The vibrations of objects produce compression waves in the surrounding air. In an enclosed space, the compression waves will reflect off surfaces and objects, and the waves produced by different objects will interact with each other to alter the spectral composition of the wave in determinate ways, with the result that the local disturbance of the air at any place will carry information about any number of objects and events, and about the environment in which they occur. This local disturbance of the air is what is detected by our ears.

\footnotetext{
${ }^{23}$ The vibration of a plucked string is actually made up of the odd harmonics of the fundamental unlike the vibration of a string excited in some other way, which includes both odd and even harmonics.

${ }^{24}$ It is in virtue of this that we can distinguish the intensity of a sound (its loudness) from the apparent force of the impact that produced the sound.
} 
The auditory system detects the frequency components that make up the complex vibrations of the soundwave that reaches the ears. To tell us about the sources of sounds it must construct a representation of objects producing sounds by extracting the information about them embodied in the pattern of frequency components detected by the ears.

If the sounds we heard were only ever produced by one object at a time the fact that a soundwave is made up of many frequency components would be unproblematic: components that are detected simultaneously would have been simultaneously produced by a single event, and successively detected components would have been produced by temporally successive parts of that event. Often, however, there are many different objects producing sounds simultaneously, so the compression wave that is detected by the ears is, at any moment, the result of the additive combination of the compression waves produced by all the sound producing events occurring in our immediate environment; as a result this compression wave is composed of frequency components produced by different objects.

Auditory perception therefore requires perceptual processing much like that involved in visual perception. We can think of the frequency components detected by the ears as analogous to the pattern of light detected by the retinas of the eyes. Just as we see things in virtue of detecting a pattern of light on a surface (the retina), so we hear things in virtue of detecting properties of soundwaves disturbing a surface (the basilar membrane). We don't, of course, see the pattern of light: our visual experience is the result of perceptual processes to which the pattern of light detected by the retina is one of the inputs. Similarly, we don't hear the frequency components of soundwaves detected by the ears; our auditory experience, including the sounds we hear, is a result of perceptual processes, to which the frequency components of soundwaves are one of the inputs. This perceptual process involves at least the following three stages.

The first stage is sensory transduction or detection: the ears detect properties of the soundwave - the local disturbance of the air. The result of this sensory transduction is, in effect, a temporal spectrogram of the soundwave which encodes the frequency and temporal properties of the soundwave's vibration. The ears detect each of the frequency components (within a detectable range) present in the soundwave's vibration. 
The second stage involves grouping together the frequency components that have been produced by the same source. ${ }^{25}$ Information about objects and events is embodied in the relationships amongst the frequency components produced by an object's vibration and the way those frequency components change over time. In order both to determine how many objects are producing sounds at any time and to extract information about those objects the auditory system must organise frequency components into groups corresponding to the objects that produced them. Frequency components need to be grouped so that all the frequency components produced by a single source are treated together, and those from different sources treated as distinct by subsequent processes. There are two kinds of grouping.

Firstly, frequency components produced at a time must be grouped together as having been produced simultaneously by a source ${ }^{26}$ secondly, simultaneous groups must be sequentially grouped over time as having been produced by a temporally extended event involving a single object, and series of such sequences grouped as having been produced by a series of events involving a single object. In order, for example, for the auditory system to determine how many objects are producing sounds at any time, it must group the frequency components it detects at a time according to the object that produced them. If the auditory system detects sequences of frequency components then grouping them together as having been produced by a single object allows information about that object to be recovered: information about how the object is changing or moving, for example; and it allows events to be recognised. In order to recognise an object as dropped onto a hard surface and bouncing the auditory system must group the sequences of frequency components produced by the object as having been produced by a single object; similarly, in order to recognise water filling a glass we must experience a single continuous sound - the auditory system must group earlier and later frequency components as parts of the same group - as produced by an ongoing process. In both cases, in order to perceive

\footnotetext{
${ }^{25}$ What follows draws on psychological accounts of auditory scene analysis, especially the work of Bregman (1990). Bregman's account explains why we experience a segregated auditory 'scene' of distinct sounds, and he shows how the auditory system organises frequency components in ways that in fact correspond to the events that produced them. He does not, however, discuss the auditory perception of the objects that are the sources of sounds, and doesn't draw the conclusion that auditory perception is object perception rather than (simply) sound perception.

${ }^{26}$ In what follows, when I talk of simultaneous grouping I mean grouping of components that were produced simultaneously; I am not implying that the process of grouping is simultaneous.
} 
the sources of sounds and their properties the auditory system must simultaneously and sequentially group the frequency components produced by that source.

How does the auditory system determine which frequency components to group together? In the case of simultaneous grouping, there are relationships that exist between components produced by the same source that are unlikely to exist between components produced by different sources. For example, an object's vibration often has frequency components that are harmonics of a fundamental frequency and so the frequency components of a soundwave that are produced by the same source will often be harmonically related. Such harmonic relationships are unlikely to exist between frequency components produced by distinct sources since it is unlikely that two simultaneously occurring natural events produce overlapping sets of harmonics. This means that if the auditory system detects a number of frequency components that are harmonically related then they are likely to have been produced by the same source. Similarly, the soundwave produced by a single event will have frequency components that share temporal properties - all the components will begin at the same time - are likely to be in phase with one another, and are likely to change over time in both amplitude and frequency in similar ways. Components produced by distinct sources are very unlikely to be related to each other in these ways. When the auditory system detects these relationships between components it groups them together and treats them as having been produced by the same source. Components that are not related in this way are not grouped together.

Just as for simultaneous grouping, there are relationships between frequency components produced by the same source at different times that are unlikely to exist between components that are produced by different sources. For example, sets of components with the same spectral composition at different times are unlikely to have been produced by different sources. Sources can change in size and so the frequency components they produce can shift in frequency. When this happens the overall pattern of frequencies is likely to remain the same. If the auditory system detects frequency modulated sets of components with the same spectral composition then they are likely to have been produced by an object that is changing. Objects cannot change instantaneously, so if successive frequency components differ greatly in frequency they are likely to have been produced by distinct sources. If two identical sets of components separated by a gap are detected, then it is more likely that they are produced by a single object than by two different objects. These examples are of 
bottom-up or stimulus driven grouping. It is likely that some grouping of sequences of components is top-down. That is, some sequences are grouped because they fit into a perceptual pattern that the auditory system recognises as likely to have been produced by a certain kind of source. ${ }^{27}$ Grouping sequences of familiar sounds produced by machines is likely to be the result of such top-down grouping.

In general the auditory system makes best sense of the frequency components it detects, where making best sense of frequency components means grouping them both simultaneously and sequentially - in such a way that they correspond to the sources that would best explain their occurrence. It is an important consequence of this that we cannot explain why the auditory system groups the frequency components that it detects in the way it does other than in terms of a process that functions to extract information about the objects that produced those frequency components. This is true of both simultaneous and sequential grouping. The auditory system groups together all and only frequency components that are likely to have been produced by the same source.

The third stage of processing involves extracting information from the frequency component groupings. ${ }^{28}$ The grouping process results in sets of frequency components that are treated by subsequent processes as having been produced by a single source. These sets of components carry information about those sources, and the fact that we can perceive various properties of the sources of sounds means that the auditory system must extract that information. Exactly what information is extracted and how it is extracted is still, for the most part, unclear. We can perceive how many sources there are, and often where they are; we can perceive various features of sources; and are able to recognise sources as events of certain kinds or involving certain kinds of object. The information extracted must be sufficient to explain these capacities. Recognition processes might match representations of the features of sources with representations of kinds of events and objects, or they might simply track some characteristic pattern of frequency components produced by certain kinds of events and objects. However exactly the information extraction and object

\footnotetext{
${ }^{27}$ Bregman calls this 'schema-based organisation': it involves 'the activation of stored knowledge of familiar patterns or schemas in the acoustic environment’ (Bregman, 1990, p. 397).

${ }^{28}$ The serial functional organisation that I am describing is an oversimplification: the results of the third stage may feed back to the second stage and alter or determine grouping. For some evidence of top-down influences on grouping, see Bey and McAdams (2002).
} 
recognition processes work, we know that they must be sufficient to explain our capacity to perceive and recognise the sources of sounds.

I have described the psychological processes involved in auditory perception; how do these processes relate to our auditory experiences and in particular our experiences of sounds and auditory objects? In virtue of their operation we experience both sounds and their sources. What sounds we experience and how we experience them to be is determined by the way the auditory system simultaneously groups the frequency components it detects: the sounds we hear correspond to simultaneous frequency component groupings. If the auditory system groups the components it detects into a single group then we experience a single sound; if it groups them into two groups, then we experience two sounds. Given that the auditory system groups frequency components that are likely to have been produced by the same source, the sounds we experience normally correspond to their sources - to the things that produced them.

What is true of sounds at a time is also true of sounds over time: the way we experience sounds as grouped is determined by the way the auditory system sequentially groups frequency components. If the auditory system sequentially groups a sequence of frequency components then we experience the corresponding sounds as grouped; we experience a sequence of sounds as grouped in virtue of the auditory system having sequentially grouped the corresponding sets of frequency components.

What sounds and auditory objects we experience is determined by the way the auditory system groups the frequency components it detects; we can only explain why the auditory system groups frequency components as it does in terms of a process that functions to tell us about the sources of those sounds and auditory objects; therefore, we can only explain why we experience the sounds and auditory objects we do in terms of a process that functions to tell us about their sources. ${ }^{29}$

\footnotetext{
${ }^{29}$ On this view of auditory perception, sounds just are a certain pattern or structure of frequency components, and an experience of a sound represents a pattern or structure of frequency components instantiated by the soundwave that is detected by the ears. An experience of a sound is veridical just in case it is produced by the pattern or structure of frequency components that would normally produce that experience; it is not veridical if it is not produced by any such pattern or if it is produced by a pattern that would not normally produce that experience. There is not space to defend this claim in detail, but it's worth noting how my view contrasts with an alternative. According to the alternative sound sources produce sounds; auditory perception functions to perceive sounds; we can tell things about the world on the basis of perceiving sounds and in virtue of our knowing what causes certain
} 


\section{Explaining auditory grouping}

The auditory system functions to represent sounds and auditory objects that correspond to their sources (to the objects and events that produced them) as part of a process that extracts information about those sources. As a result our experience represents both sounds and the sources of sounds, and we normally experience sounds that correspond to their sources.

This account of the function of auditory perception provides an explanation of our experience sounds as grouped - an explanation of why we experience auditory objects. We experience sounds as grouped as the result of a process that functions to tell us about the sources of sounds. Sequential grouping is a necessary step in the process of extracting the information about sound sources - in particular, about events involving them - that enables us to perceive and recognise them.

How does this explanation in terms of function relate to the explanation in terms of the purely auditory principles of grouping? To the extent that the principles of grouping are true generalisations about experience, the explanation in terms of the function tells us why those principles hold. They hold because sounds grouped in accordance with the principles are likely to be grouped in a way that corresponds to their sources. For example, the pitch a sound is experienced to have is determined by the fundamental frequency of the object vibration that produced it. ${ }^{30}$ The fundamental frequency of an object's vibration is determined by the size and shape of the object. It is unlikely that two identical naturally occurring objects produce sounds in the same period of time; therefore, a sequence of sounds all of which have the same pitch are likely to have been produced by a single object. The pitch of sounds produced by an object can change only if, and as fast as, the object changes. A sequence of sounds all of which have closely related pitches may have been produced by a single object that is changing in size; but two sounds with very different pitches are unlikely to have been produced by the same object. Sounds grouped according to the proximity of their pitches will, therefore, be grouped in a way that is likely to correspond to their sources. Most naturally occurring sounds have timbre determined by the complex

kinds of sounds. Given the way auditory perception actually functions, this alternative cannot be right. For a more detailed discussion, see Nudds (forthcoming).

${ }^{30}$ This is somewhat simplified, but not in a way that affects the argument. 
spectral composition of frequency components that determine them. The vibrations of two naturally occurring objects are very unlikely to have exactly the same spectral composition; therefore, a sequence of sounds all of which have the same spectral composition or timbre are very likely to have been produced by the same object and two sounds with different timbres are very unlikely to have been produced by the same object. Sounds grouped according to their timbres will, therefore, be grouped in a way that is likely to correspond to their sources.

Explanations of grouping in terms of the principles of grouping are therefore consistent with explanations in terms of the function of auditory perception, but explanations in terms of function are better explanations because they tell us why the principles are true. Furthermore, although the principles of grouping may be consistent with explanations in terms of function, and may describe generalisations that are true of experience, it doesn't follow that the auditory system uses or follows the principles in determining how to group frequency components. It is unlikely that the auditory system groups sounds because they are similar in pitch or similar in timbre - it is unlikely, that is, that those sensory qualities of sounds are causally explanatory of grouping. It is more plausible to suppose that the auditory system groups sets of frequency components because they have the same spectral composition, and that those sets of frequency components with the same spectral composition determine experiences of sounds with the same timbre. The consequence of that would then be that similar sounds are grouped together, as described by the principles. If that's right, the truth of the principles of grouping is a consequence of the way the auditory system functions, but is not explanatory of its function.

\section{What are auditory objects?}

The point of this account of auditory function is that it doesn't just tell us in what circumstances we experience a sequence sounds as grouped, it explains why we experience them as grouped in those circumstances; it therefore provides the basis for an account of what our experience of auditory object is of, and what our experience represents auditory objects as being.

I have argued that auditory experience represents both sounds and the sources of sounds. How do we experience the connection between the sounds we experience and the sources of those sounds? We don't experience the source of a sound 
independently of experiencing the sound that it produces. When we experience a sound we experience it as apparently having been produced a source of a certain kind. For example, in experiencing the sound produced by a solid object falling onto a hard surface we experience a sound as apparently having been produced by a solid object falling onto a hard surface; in experiencing the sound made by a bird singing outside the window we experience a sound as apparently coming from outside. Normally, when we hear a sound we hear it as having been produced by a source; in virtue of that we can hear the source. That we hear sounds as produced by their sources is reflected in the way we describe sounds: we talk of the sound of a dropped ball and of a bird singing. Describing a sound as the sound of something can be naturally understood to mean the sound made by or produced by that thing.

Sounds are produced by sources: a sound has the property of having been produced by a source of a certain kind. When we experience a sound as having been produced by a source, our experience represents it as having that non-intrinsic property. Therefore, our auditory experience represents sounds and the sources of sounds and it represents sources as the sources of sounds by representing sounds as having a non-intrinsic property - the property of having been produced by a source of a certain kind. We can perceive sounds as having been produced by their sources in virtue of our experience (veridically) representing them as having been so produced. ${ }^{31}$ As well as offering the best explanation of our experience of sounds and their sources, this description is consistent with the fact that our auditory system functions to extract information about the objects and events that produce the soundwaves it detects. It is because our auditory experience represents sounds as produced by their sources that we can attend to our auditory experience in the two ways that I described earlier. When we hear a glass bottle dropping onto the floor and bouncing our experience represents the sounds made by the bottle hitting the floor and bouncing, and it represents them as produced by an event of a certain kind. We can attend either to the sounds - to how many they are, how loud they are, their pitch and rhythm - or we can attend to the event represented by our experience: an object bouncing on a hard surface.

\footnotetext{
${ }^{31}$ Note that the explanation of what makes an experience of a sound veridical is different to the explanation of what makes an experience of the source of a sound veridical. That means that an experience may veridically represent a sound, but misrepresent the source of that sound. There are a number of auditory illusions that should be explained in just this way.
} 
This account of our experience of sounds as produced by their sources can be extended to include our experience of sequences of sounds (which include continuous sequences not composed of discrete sounds). The sounds that compose a sequence of sounds may be experienced as produced by a number of distinct sources or as produced by the same source. When they are experienced as having been produced by the same source - as all having come from the same object - they are experienced as related to one another - as grouped together. Each sound of a sequence of grouped sounds is experienced as having been produced by a source; they are experienced as grouped in virtue of being experienced as having been produced by the same source. We can explain, therefore, what it is to experience a sequence of sounds as grouped as being an experience of them as having been produced by a single source. A sequence of sounds not experienced as grouped are experienced as having been produced by different or distinct sources.

This claim is plausible given the account that I have given of the function of auditory perception. Auditory perception functions to tell us about the sources of sounds; we experience sounds as grouped together as the result of a processes that function to extract information about their source, and so we experience them as grouped because they are produced by the same source (or do so if the auditory system is functioning normally). In virtue of the operation of the processes that extract information about the source of a sequence of sounds, we have an experience that represents both the source of the sounds and the sequence of sounds that it produces. My claim is that we hear the sequence of sounds as having been produced by the source we hear. When we hear the sounds made by footsteps, for example, we hear them as having been produced by a single object; when we hear an object as bouncing we hear a sequence of sounds produced by a single object. In both cases, we hear the sequence as a grouped sequence because we hear them as produced by a single object. If our experience didn't represent those sequences of sounds as having been produced by a single object then we wouldn't experience footsteps as footsteps, or an object bouncing as an object as bouncing, we would simple experience a sequence of unrelated sound producing events.

Auditory experience represents sounds as apparently produced by a source of a certain kind; that is, with certain properties. For the experience to be veridical the sound must have actually been produced by a source of that kind. This has the implication that our experience of sounds normally commits us to the existence of 
something other than sounds. That is surely right. Suppose that you hear the sound of a drum apparently being played in middle of the room. Your experience tells you that there is something happening there, that an event of a certain sort - the playing of a drum - is occurring. If there is no drum there, your experience has misled you. The experience wouldn't be veridical even if we contrived - using an array of speakers, for example - to reproduce exactly the sounds that a drum being played there would make. An experience produced in this way would be no more veridical than would be the visual experience of a perfect hologram of a vase on a table in front of you. A visual experience produced by a perfect hologram does not represent the world as it really is: it represents the existence of an object - a vase - that doesn't exist. Similarly, an auditory experience of a drum playing represents the existence an object; if there is no object being played there then your experience has misled you. It is because our auditory experience of sounds commits us to the existence of objects other than sounds that surround-sound systems in the cinema are so effective. Such systems use sounds to create the illusion of objects moving or being located around the listener. When you hear such sounds it seems as if objects really are moving past and around you. Knowing that the experiences are not veridical does not alter the effect: knowing that there are in reality no objects flying past does not prevent it seeming as if there are objects flying past. That it seems that there are objects flying past when we know that there aren't indicates that the illusion is perceptual and not the result of a judgment made on the basis of the experience. ${ }^{32}$

The claim that auditory experience represents sounds as having been produced by their sources can seem puzzling if we think that perceptual experience is restricted in what properties it can represent to those properties that determine how things perceptually appear. ${ }^{33}$ Since having the property of being produced by a source of a certain kind is not a matter of a sound's having a certain appearance, how does our experience represent it as having that property? And since nothing other than sounds can auditorily appear to us, how can our auditory experience represent anything other

\footnotetext{
${ }^{32}$ The illusion shows the immunity to judgment that is characteristic of the content of an experience as opposed to the content of judgement.

${ }^{33}$ There are two conceptions of appearance that are relevant here. Something can appear F if, taking our experience at face value, we would judge that it is $\mathrm{F}$ or something can appear $\mathrm{F}$ if it has the sensory quality of F-ness. Sometimes talk of appearance is shorthand for how someone would judge something to be; sometimes it stands for 'sensory' appearance. In the following discussion I mean it in the second sense.
} 
than sounds? In particular, how can it represent the objects that are the sources of sounds?

If we think that perceptual experience is restricted to representing those properties that determine how things perceptually appear then our visual experience of objects can seem similarly puzzling. We see solid objects as solid objects and not just as the facing surfaces of solid objects, but how can visual experience represent something as actually being, say, cubic - as something with a rear surface - rather than merely having the appearance of being cubic - as a surface with the same appearance as that of a cube ${ }^{34}$ In representing something as cubic our visual experience represents it as having properties that go beyond the properties that actually determine how it appears.

Peacocke (1993, p.169) has claimed, surely correctly, that we experience objects as specifically material objects: a visual experience of a boulder in front of you produced by a perfect hologram of a boulder does not represent the world as it actually is, even if the hologram is visually indistinguishable from a real boulder. The content of the experience goes beyond the representation of the boulder's appearance - it represents the boulder as a material object; that is, as having the properties and causal powers that are essential to something's being a material object. Peacocke suggests that we can explain how someone can have a perceptual representation of a material object by supposing that their experience serves as input to a (perhaps only implicitly known) theory - an intuitive mechanics - whose theorems give content to their concept of a material object. Whether or not we accept the details of Peacocke's account, he is certainly right about two things. First, that visual experience represents objects as having properties that are not properties that determine how the object visually appears; and second, that an explanation of how visual experience can have such content will appeal to more general capacities of the subject - such as an intuitive understanding of mechanics - that are not perceptual capacities. What is true of the content of visual experience is also true of the content of auditory experience, and so whatever explanation we give of how visual experience can have content that represents material objects will also apply to auditory experience. The claim that auditory experience represents sounds as having been produced by their sources is,

\footnotetext{
${ }^{34}$ Since a solid cube can be visually indistinguishable from the facing surface of a cube - a cube from which every part not visible from the subject's point of view have been removed - having a rear surface and not being hollow are not properties that contribute to the appearance of a solid cube.
} 
therefore, no more puzzling or problematic - and so no more objectionable - than the claim that visual experience represents objects as material objects.

What are our experiences of auditory objects experiences of? An auditory object is a sequence of sounds which are such that they are normally experienced as having been produced by a single source. Normally - when the auditory system is functioning properly - the experience of an auditory object is the experience of a sequence of sounds that have been produced by a single source. In such circumstances, our experience veridically represents the sounds as having been produced by a single source. Some sequences of sounds, however, which are such that they are normally experienced as having been produced by a single source, have not been produced by a single source. This is most likely to happen when the sounds are produced in a way that is, from the point of view of the function of the auditory system, abnormal. In such circumstances the auditory system groups sounds that have not in fact been produced by a single source, and the resulting experience misrepresents those sounds as having been produced by a single source. When this happens we have an experience of an auditory object that is composed of sounds that are not all produced by a single source, but merely seem to be.

I began with a description of auditory grouping as it occurs in a passage of music, but the subsequent discussion has concerned ecological rather than musical sounds. How does the account I have given of auditory grouping apply to our experience of grouping in music? We perceive music in virtue of the operation of the same mechanisms that enable us to perceive the sources of sounds. The auditory system treats the sounds produced during musical performances as if they had been normally produced by ecological events involving natural objects. Our experience of musical sounds and of auditory objects is the result of the auditory system making the best ecological sense of the frequency components it detects. In fact, such sounds are produced abnormally. ${ }^{35}$ The resulting experience of auditory objects - of groups of sounds that constitute melodies - may be of sounds that only seem to have a single source; but since in listening to music we adopt a 'musical' or 'acousmatic' attitude to the sounds, that rarely matters to us.

\footnotetext{
${ }^{35}$ From the point of view of the function of the auditory system, musical sounds are produced in an abnormal way: naturally occurring objects and events - the kind of objects and events that the auditory system evolved to perceive - are very unlikely ever to produce the patterns of frequency components produced by groups of musical instruments playing in harmony. The resulting experience of musical sounds may have little or no ecological significance.
} 
Although we can explain our experience of music within the general framework of auditory perception that I have described, it would be a mistake to think of it as paradigmatic of auditory perception; it is, rather, the consequence of the operation of a perceptual system whose function is far more mundane. ${ }^{36}$

\section{References}

Bey, Caroline, and Stephen McAdams. 2002. Schema-based processing in auditory scene analysis. Perception and Psychophysics 64 (5):844-854.

Bregman, Albert S. 1990. Auditory scene analysis: the perceptual organization of sound. Cambridge, Ma.: MIT Press.

Bregman, Albert S, and J. Campbell. 1971. Primary auditory stream segregation and perception of order in rapid sequences of tones. Journal of Experimental Psychology 89: 244-249.

Carello, C., J. B. Wagman, and M. T. Turvey. 2005. Acoustic specification of object properties. In Moving image theory: Ecological Considerations, edited by J. D. Anderson and B. Fisher. Carbondale, IL: Southern Illinois University Press.

Deutsch, D. 1999. Grouping mechanisms in music. In The psychology of music, edited by D. Deutsch: Academic Press.

Freed, D. J. 1990. Auditory correlates of perceived mallet hardness for a set of recorded percussive events. Journal of the Acoustical Society of America 87:311-322.

Gaver, W. W. 1993. How do we hear in the world? Explorations in ecological acoustics. Ecological Psychology 5:285-313.

Kunkler-Peck, A., and M. T. Turvey. 2000. Hearing shape. Journal of Experimental Psychology: Human Perception and Performance 1:279-294.

McAdams, Stephen. 1993. Recognition of sound sources and events. In Thinking in Sound, edited by S. McAdams and E. Bigand. Oxford: Oxford University Press.

Michotte, A. 1963. The Perception of Causality. Translated by P. Heath. New York: Basic Books.

\footnotetext{
${ }^{36}$ I would like to thank to Roberto Casati and an anonymous referee for comments. Research for this paper was supported by an AHRC research leave award.
} 
Neuhoff, John. 2004. Auditory motion and localisation. In Ecological Acoustics, edited by J. Neuhoff. London: Academic Press.

Nudds, Matthew. Forthcoming. Auditory Perception and Sounds.

Peacocke, C. 1982. Sense and Content. Oxford: Clarendon Press.

- 1993. Intuitive mechanics, psychological reality and the idea of a material object. In Spatial representation: problems in philosophy and psychology, edited by N. Eilan, R. A. McCarthy and B. Brewer. Oxford: Blackwell Publishers.

Peretz, Isabelle. 1993. Auditory agnosia: a functional analysis. In Thinking in Sound, edited by S. McAdams and E. Bigand. Oxford: Oxford University Press.

Russell, M., and M. T. Turvey. 1999. Auditory perception of unimpeded passage. Ecological Psychology 11:175-188.

Schiff, W., and R. Oldak. 1990. Accuracy of judging time to arrival: Effects of modality, trajectory, and gender. Journal of Experimental Psychology: Human Perception and Performance 16:303-316.

Scruton, Roger. 1997. The Aesthetics of Music. Oxford: Oxford University Press.

VenDerveer, N. J. 1979. Ecological acoustics: Human perception of environmental sounds, PhD thesis, 1979. Dissertation Abstracts International, 40, 4543B. (University Microfilms No. 80-04-002).

Warren, Richard M. 1999. Auditory perception: a new analysis and synthesis. Cambridge: Cambridge University Press.

Warren, W. H., and R. R. Verbrugge. 1984. Auditory perception of breaking and bouncing events: A case study in ecological acoustics. Journal of Experimental Psychology: Human Perception and Performance 10:704-712.

Wildes, R., and W. Richards. 1988. Recovering material properties from sound. In Natural computation, edited by W. Richards. Cambridge, MA: MIT Press. 